\title{
Impacto que genera un proyecto sobre oratoria en estudiantes de primaria de zonas rurales de Cartago
}

\author{
The Impact an Oratory Project generates to Primary \\ School Students who live in Rural Areas of Cartago
}

María Gabriela Amador-Solano'

Fecha de recepción: 10 de junio del 2013

Fecha de aprobación: I de octubre del 2013

Amador-Solano, M. Impacto que genera un proyecto sobre oratoria en estudiantes de primaria de zonas rurales de Cartago. Tecnología en Marcha. Número Especial. Pág 94- 104. 


\section{María Gabriela Amador Solano}

Nació en San José el 26 de setiembre de 1977. Cursó la primaria en la Escuela Central de Tres Ríos y la secundaria en el Colegio Sagrado Corazón de Jesús, en Cartago. En ambas instituciones se graduó con mención honorífica.

En 1995 ingresó a la Universidad de Costa Rica, a la carrera de Bachillerato y Licenciatura en la Enseñanza del Castellano y Literatura, y a la carrera de Filología Española. En el año 2003 se graduó en la Maestría Enseñanza del Español como Segunda Lengua. Cinco años después obtuvo otra especialidad en Aplicaciones didácticas en la Enseñanza-Aprendizaje de Lenguas, de la Universidad Internacional Iberoamericana de Puerto Rico. En el año 2006 se graduó como Técnica en Administración de Empresas, del Instituto Tecnológico de Costa Rica. Actualmente está realizando las gestiones para ingresar a un Doctorado en Educación con énfasis en Tecnología Educativa.

En sus años universitarios, trabajó como asistente becada del CIGEFI, ubicado en la Universidad de Costa Rica, donde tuvo que investigar en el tema de Meteorología y Física. También participó como filóloga en la Revista Ingeniería de la Universidad de Costa Rica. En 1998 comenzó a impartir lecciones particulares de Español a estudiantes de colegio. Desde entonces y hasta el año 2004, estuvo preparando estudiantes para exámenes de convocatoria y para las pruebas de Bachillerato en Educación Media. Trabajó por más de 5 años en diferentes colegios de secundaria tales como: Colegio Jorge Volio Bilingüe, Colegio Miravalle Bilingüe, Colegio El Rosario y Colegio Sagrado Corazón de Jesús; así como en Institutos de Enseñanza del Español como Segunda Lengua.

Fue profesora de la Universidad Estatal a Distancia, de los cursos de Lengua y Literatura. Además, desde el año 2003 ofrece cursos en la Universidad de Costa Rica, en Estudios Generales y en la Facultad de Educación. A partir del año 2004, obtiene su propiedad en el Instituto Tecnológico de Costa Rica, en la Escuela de Ciencias del Lenguaje, específicamente ofrece los cursos de Comunicación Escrita,
Comunicación Oral, Comunicación Educativa, Comunicación Técnica y Seminario de Investigación. También imparte Centros de Formación Humanística relacionados con áreas de Cultura y Literatura. Fue profesora de la Universidad EARTH, y gestora del Programa de Español para extranjeros, en esta misma casa de enseñanza.

La profesora Amador tiene más de 10 años de experiencia como docente universitaria, por ende, ha realizado diversos trabajos de investigación de gran impacto para la comunidad. Es investigadora responsable del proyecto de extensión Plan piloto para la capacitación de expresión oral de maestros y maestras de primaria de I y || Ciclo, de zonas rurales del cantón central de Cartago.

También ha participado en Congresos Nacionales e Internacionales con ponencias sobre temas de Expresión Oral, Formas de tratamiento en la interacción entre hablantes nativos y hablantes extranjeros que estudian en la provincia de Cartago, Actividades que motivan el aprendizaje del léxico en la enseñanza del español como segunda lengua, Análisis de los textos literarios que se incluyen en el tercer ciclo de secundaria como parte de una propuesta que motive la lectura de los educandos. Se ha presentado en Guatemala, El Salvador, Nicaragua y Costa Rica.

María Gabriela se describe como una profesional que ama la docencia, le apasiona dar clases, participar de proyectos de ayuda social comunitaria. Tiene una sólida experiencia en la enseñanza del español como segunda lengua y en temas de educación como currículo, pedagogía, psicología del estudiante y didáctica general. Afirma tener capacidad para liderar proyectos, ejemplo de ello es la coordinación del Técnico en la Enseñanza del Español como Segunda Lengua, donde debe dirigir y controlar labores tanto académicas como administrativas. Le encantan los retos, las nuevas oportunidades y el trabajo responsable. Afirma tener una motivación especial cada día para desempeñarse como profesora, coordinadora, compañera, esposa, hermana e hija; esto es su fe y agradecimiento a Dios. 


\section{Palabras clave}

Oratoria; enseñanza primaria; expresión oral; capacitación; disertación.

\section{Resumen}

Este artículo propone evidenciar el desarrollo e importancia de aplicar un proyecto de extensión en siete escuelas rurales del Circuito 05 del cantón central de Cartago. El objetivo es fomentar las bases idóneas para el buen desarrollo de la expresión oral desde edades tempranas en la enseñanza primaria. El proyecto fue diseñado como taller de capacitación para maestros y maestras de las escuelas seleccionadas, con el fin de que lo implementen con sus alumnos y alumnas a través de un club de oratoria. Las investigadoras pudieron observar el impacto que generó y el interés en cada centro educativo, a través de las disertaciones de los niños y las niñas. Se diseñó un planeamiento didáctico que integra objetivos, contenidos, actividades, evaluación y observaciones, para que se aplique según la necesidad de cada institución.

\section{Key words}

Oratory; elementary school; oral expression; training workshop; dissertation.

\begin{abstract}
This article aims at showing the development and relevance of an extension project in seven rural schools located in "circuito 05" in the central area of Cartago. The main goal is to enhance oral communication in elementary school students. The project was designed as a training workshop for the teachers in the chosen schools in order to be taught to students by implementing an oratory club. In each student's dissertation, the researchers observed the enthusiasm that the project caused in the schools. Objectives, contents, activities, assessment and observations were designed in a didactic plan to be used upon needs of institutions.
\end{abstract}

\section{Introducción}

Los humanos tenemos el privilegio de poder expresarnos a través de la palabra. La sociedad actual exige un alto dominio de la comunicación, tanto escrita como oral. La base educacional que se enseña desde la etapa primaria es elemental para motivar el desarrollo de las habilidades lingüísticas, las cuales le darán al individuo herramientas para defenderse con seguridad en cualquier contexto de socialización. De ahí la necesidad de presentar un proyecto de extensión a la comunidad, con el fin de ofrecer a las escuelas rurales que más lo requieren una capacitación sobre oratoria moderna.

Las deficiencias en el manejo de la expresión oral se notan en nuestros estudiantes universitarios. Cada uno de los niveles de educación culpa al precedente: la universidad a la educación secundaria y la secundaria a la primaria. Es primordial considerar la capacitación de la comunicación oral desde la base inicial en la escuela, así como reforzarla en la secundaria y estimular su práctica en la universidad. Todo esto a través de actividades didácticas que cumplan con el objetivo de repasar con ejercicios las particularidades de una buena disertación.

Dada la necesidad de capacitación de la comunidad, dos profesoras de la Escuela de Ciencias del Lenguaje del Instituto Tecnológico de Costa Rica (ITCR) decidieron plantear un proyecto de extensión que busca capacitar a los maestros y maestras de primaria, para que estos transmitan el conocimiento y motiven a sus estudiantes a adquirir las herramientas que les permitan prepararse para expresarse oralmente. Se analizaron los aportes que tiene el enfoque comunicativo para el diseño de un plan de capacitación, que permita implementar en cada centro educativo un club de oratoria. Para ello, se diseñaron los materiales y estrategias metodológicas que mejor respondieron al seguimiento preciso que debe tener el docente cuando prepara a sus educandos. 
Por otro lado, una de las razones fundamentales de la ejecución de este proyecto es la convocatoria que hace la Municipalidad de Cartago en agosto de cada año a todos los estudiantes que deseen participar en el Festival de Oratoria, actividad que forma parte del programa de celebración de la Semana Cívica Cultural en conmemoración del aniversario de la independencia nacional. Para ello, se contemplan los centros educativos del cantón central de Cartago, cada escuela tiene la oportunidad de nombrar a tres representantes máximo por categoría, esto es: I y || Ciclo y III Ciclo. Los estudiantes se inscriben y presentan su exposición oral de acuerdo con una temática que escogen y desarrollan, ya sea con ayuda que reciben en sus instituciones educativas, a través de profesores externos o, en otros casos, solamente con la asesoría de sus padres.

La experiencia en este concurso permitió analizar la escasa cantidad de estudiantes que proceden de zonas rurales, a diferencia de la participación de las escuelas del centro de la provincia, que tienen mayor acceso a la capacitación de docentes. Sin embargo, independientemente de la procedencia de los educandos, se nota en general que carecen de la preparación adecuada en aspectos como la utilización de la voz, la estructuración de la exposición y el uso de la expresión corporal. Además, su experiencia se resume en aprenderse de memoria la exposición del tema asignado.

\section{Una idea de proyecto más que un compromiso}

Los educadores del ITCR tienen un compromiso social con la comunidad. Se trata de realizar proyectos de extensión que generen resultados positivos ante las necesidades frecuentes. Cartago es una provincia que poco a poco se da a conocer por sus proyectos de bien social, las personas responden para mejorar su formación y actualizarse en diferentes campos. De ahí la motivación de las capacitadoras por un proyecto que pretendió diseñar los materiales idóneos y las estrategias metodológicas para la creación de un taller de oratoria en cada una de las escuelas escogidas.

La selección de las escuelas participantes fue guiada por el lugar en que están ubicadas, esto es, escuelas rurales o en la periferia del cantón; así como de acuerdo con la capacidad socioeconómica que tienen los estudiantes. Además, específicamente los niños y niñas de estas escuelas no cuentan con docentes especialistas en Comunicación Oral que les impartan los talleres. Las instituciones educativas participantes en este proyecto pertenecen a la provincia de Cartago: Escuela Filadelfo Salas Céspedes en Lourdes, Centro de Atención Prioritaria Cocorí de Aguacaliente, Escuela Proyecto Manuel de Jesús, Escuela Antonio Camacho Ortega de Corralillo, Escuela de Quebradilla, Escuela Alto de Quebradilla y Escuela Sixto Cordero Martínez de Quebradilla.

Otra variable que se tomó en cuenta para la elección de los centros educativos fueron los recursos, pues a diferencia de muchas que están en el centro del cantón, estas escuelas no tienen los recursos suficientes para capacitar a los estudiantes en estos aspectos de oratoria. Cabe resaltar que este plan piloto busca que otras escuelas tomen la iniciativa de solicitar este taller para que también se beneficien de esta experiencia inicial.

\section{Propuesta teórica}

Con el fin de diseñar un plan de capacitación, se investigaron los diferentes teóricos para analizar cada una de las propuestas que se pueden adaptar mejor a la población meta del proyecto. Antes de diseñar los talleres, fue necesario seleccionar la base teórica sobre conceptos fundamentales como definición de oratoria, motivación al exponer, estructura de una disertación y elementos de una buena elocución tales como: pronunciación, mirada, control del cuerpo y actitud hacia el auditorio. Además, los propósitos de la comunicación, el entrenamiento sistemático, los ejercicios de relajación y actividades para estimular la expresión oral.

A continuación se expone un resumen de los conceptos y teoría específica que sirvieron de base para la creación de los talleres de capacitación, dirigidos a niños y niñas de I y I| ciclo de escuelas rurales de la provincia de Cartago.

Primeramente, es necesario referirse al concepto de oratoria, el cual tiene sus orígenes en la antigüedad clásica. El Diccionario de la Real Academia Española define el término como el "Arte de hablar con elocuencia" (Real Academia Española), es decir, saber cómo comunicarse a través de la palabra con pertinencia y eficacia. Por otro lado, Briz (2008: 20) establece que los principios retóricos son una parte 
también de lo que hoy se entiende por saber hablar en público, la tarea de una persona que se presenta y utiliza el lenguaje ante otro u otros individuos con un fin determinado.

El orador es el creador del mensaje en el proceso de comunicación. Sea cual sea el contexto, el discurso y el lenguaje del orador depende de sus experiencias, sentimientos y carácter (Verderber, 2000: 3). Es decir, la formación que tiene un niño o niña es distinta a la de un adulto, por eso la motivación que se planee para que un individuo pueda emitir su mensaje dependerá de la edad, contexto, nivel de aprendizaje, etc.

Según Spitzberg (2003), si hay motivación podrá haber cambios para perfeccionar nuestra comunicación, de lo contrario será difícil. Ese elemento fundamental tiene relación directa con la percepción de identidad, la autodefinición y autoestima, pues en la medida en que el individuo se conozca a sí mismo, así se podrá comunicar y elegir las personas con quienes se va a relacionar. De esta manera, la autodefinición influye en la forma en que se habla de sí mismo con el resto de las personas, de hecho, existirá mayor probabilidad de comunicarse de manera positiva.

Según Verderber (2000), cuando la persona debe prepararse como orador necesita formular objetivos, definir conceptos, plantear preguntas, recabar datos, organizar información, unir ideas y evaluar sus propios argumentos e información; todo esto le permite desarrollar su pensamiento crítico. Este proceso de criticidad es uno de los aspectos primordiales en la formación del educando, pues usa la lógica y el razonamiento para transmitir información.

Una buena disertación obedece a una buena elocución, esta se define como la manera de utilizar la palabra en forma oral e integra el modo de elegir y distribuir las ideas, así como los aspectos corporales. En este sentido, la emisión de la voz es fundamental. Brehler (2000: II) establece que "si se quiere lograr una correcta emisión de la voz y evitar deformaciones, se debe conocer el mecanismo que interviene en la emisión y saber controlar sus parámetros: volumen, ritmo y entonación. Además, se tiene que aplicar conscientemente la técnica apropiada, para lograr la mejor posición del sonido y una proyección más expresiva de la voz; por lo tanto es necesario desarrollar este tema mediante ejercicios y explicaciones, tanto fisiológicas como fonéticas, para fomentar una correcta utilización de la voz frente a un público.

La pronunciación adecuada de los sonidos es necesaria para que el oyente escuche bien el discurso y, sobre todo, lo comprenda. Briz (2008), en su libro Saber hablar, define la voz como el recurso sonoro y audible que proporciona la transmisión de palabras al receptor; constituye un vehículo que llevará el mensaje hasta el receptor. Pronunciar bien es una destreza cuyo dominio exige esfuerzo y aplicación de un método, se debe llegar a ser capaz de conocer y manejar el timbre de voz que se tiene, para adaptar las cualidades de la articulación vocal, estas son: volumen, velocidad, tono, pausas y flexibilidad.

Respecto al volumen, se debe usar para realzar los elementos importantes del discurso, con ello, todos los especialistas en educación de la voz, según Briz (2008), "aconsejan no centrar el esfuerzo vocal en la garganta, sino en la capacidad de aire que somos capaces de contener. Voz y respiración forman, pues, una unidad".

Sobre las pausas y la velocidad, se complementan porque la pausa funciona como mecanismo de control de la velocidad. Según Briz, la pausa ayuda a preparar lo que se va a comunicar, incluso resulta muy útil para evitar algunos vicios expresivos, como las muletillas, por ejemplo. Al mismo tiempo, la pausa sirve como mecanismo para que el público reflexione sobre lo que ha escuchado.

Por otro lado, a través del tono o altura musical, se debe modular la voz en un nivel medio, entre tonos graves y agudos. A través del tono se manifiestan el carácter del orador y su estado de ánimo: alegría, confianza, inseguridad, etc. Es necesario, para este proyecto, aprovechar la energía de los niños y niñas para que empleen tonos vivos y atrayentes en la exposición (Briz: 2008).

Finalmente, plantea este autor que la flexibilidad es la capacidad del expositor para variar las cualidades de la voz, anteriormente mencionadas. Este ejercicio debe hacerse en función del interés persuasivo que se provoque en el público, a través de la variedad de matices que se puedan usar para una buena transmisión del mensaje.

Otro aspecto importante de la elocución es la expresión corporal. El cuerpo como un todo tiene que reforzar el mensaje que se está emitiendo, no debe ser un obstáculo para que la persona se dé a 
entender. Son muchos los autores que trabajan este aspecto (Ander-Egg \& Aguilar, 2002; Barquero, 2003; Müller, 2004; Verderber, 2000) y apuntan la importancia de trabajar gestos, posturas, expresiones de la cara, mirada y vestimenta del orador, pues las deficiencias en estos aspectos pueden entorpecer el objetivo de la exposición.

Tal como señala Verderber (2000), la eficacia para comunicar lo que se tiene en mente depende en gran medida de la manera en que las acciones corporales no verbales complementan la voz. Por ejemplo, la expresión facial tiene que ver con el movimiento de la boca y los ojos, los oyentes buscan la expresión facial que parece reflejar el pensamiento del orador.

Dentro de los elementos de la expresión corporal están los dedos, las manos y los brazos. Por ejemplo, los dedos se usan de manera consciente para describir o hacer énfasis. El movimiento de los brazos tiene que ver con la acción de todo el cuerpo. Por eso, si se está concentrado en lo que se dice, probablemente la acción corporal sea la apropiada. Para mejorar la expresión corporal, Verderber propone la grabación de sí mismo, para poder autoevaluarse y así monitorear los movimientos que se producen.

El último aspecto fundamental de un buen orador es el desarrollo de su discurso. Un expositor exitoso no debe poseer solo una buena oratoria, expresión corporal o adecuada dicción sino que, además, sus ideas deben desarrollarse de manera ordenada, coherente y cohesionada. Solo así se podrá lograr una comunicación verdadera entre ambos participantes.

Según el Diccionario del uso del español de María Moliner, discurso es: "En sentido amplio, conjunto de palabras con que alguien expresa lo que piensa, siente o quiere. En sentido más restringido, exposición sobre un asunto serio hecha en tono ilustrativo por una persona a otras".

La finalidad del discurso dependerá en gran medida del tipo de modalidad empleada, las características del orador y el contexto en el que se emite. Existen varios propósitos en este tipo de comunicación, por ejemplo, entretener se refiere a buscar una respuesta positiva y agradable del público; si es informar, define claramente la comprensión de un tema; mientras que persuadir trata de motivar al auditorio para realizar acciones (Fournier, 2005).
Según Fonseca (2005), la organización comienza con una preparación del tema, que debe contemplar los intereses y necesidades del público, así como el nivel de conocimiento que se tiene. En esta etapa es importante ordenar las ideas y realizar un bosquejo que permita distribuir la introducción, el desarrollo y la conclusión, de la mano de la coherencia y el equilibrio en la cantidad de información. En síntesis, el comunicador necesita cumplir cuatro etapas básicas: I. tener secuencia lógica, 2. contar con un formato de introducción, desarrollo y conclusión, 3. tener paralelismo en su redacción, 4. lograr equilibrio en sus contenidos.

Para Verderber (2000), las respuestas a las siguientes preguntas acerca del escenario permiten la escogencia de un tópico y objetivo del discurso que se relacionen con los intereses del público. Estas son: cuáles son las expectativas especiales para el discurso, cuál es la extensión de tiempo apropiada, cuál será la dimensión del auditorio, dónde será presentado el discurso, cuándo será presentado, en qué momento del programa se da la disertación, qué equipo es necesario para presentarlo y cuáles son las características del público meta.

Para capacitar a la población de las escuelas y obtener un resultado positivo, el profesor debe guiar al estudiante a que se dé cuenta de hasta dónde puede ayudarse a sí mismo a comprender usando sus propios recursos internos, mediante la formulación de preguntas y respuestas que definan lo que desea exponer y la forma en que lo va a realizar. El niño o niña debe conocer el vocabulario que utiliza, debe investigar qué desea expresar, tiene derecho a estar en desacuerdo con un asunto mientras fundamente su posición.

Entre las estrategias didácticas de la comunicación oral es indispensable tomar en cuenta el entrenamiento sistemático de los alumnos en los niveles de identificación y selección de los aspectos más relevantes del mensaje; así como su preparación sicológica para situaciones en las que no comprendan en su totalidad lo que escuchan. Los alumnos deben aprender a interpretar lo que leen, analizar los textos literarios, buscar información en las redes tecnológicas, seleccionar la bibliografía adecuada que incluye en su disertación.

El taller de expresión oral tiene las siguientes funciones: introducir nuevo vocabulario, facilitar la comprensión de los temas, hacer que el alumno 
desarrolle destrezas comunicativas y expresivas que le permitan usar al máximo sus recursos lingüísticos y crear en los educandos un sentido de confianza en sí mismos (Bello, 1990). La confianza le dará seguridad a la hora de exponer, esto se logra mediante la constante práctica y la motivación para investigar un tema seleccionado según sus intereses o necesidades; exponer con propiedad mediante argumentos válidos y cerciorarse de que la información esté actualizada.

Richards (1990) plantea que deben seguirse dos enfoques en la enseñanza de la expresión oral:

a. Enfoque indirecto: donde la competencia conversacional es vista como el producto de aprendizajes atractivos en la interacción conversacional. Se aprende a hablar dialogando. El estudiante debe aprender a manejar turnos, así como la apertura y el cierre de la conversación.

b. Enfoque directo: consiste en el planeamiento de un programa conversacional alrededor de microdestrezas específicas, estrategias y procesos que son desarrollados en una conversación fluida.

En el caso de un estudiante de primaria, Richards (1990) plantea que este puede crear con el lenguaje, preguntar y contestar cuestiones simples o sobre temas familiares y manejar situaciones comunes. Para cumplir con las funciones específicas de la expresión oral se deben tomar en cuenta los siguientes requisitos (Bello, 1990).

I. La clase debe ser significativa a través de situaciones que tengan sentido para el estudiante. Por ejemplo, las mismas frases o preguntas durante diversas situaciones pueden ser significativas o volverse solo ejercicios de práctica formal.

2. El planeamiento de la clase debe tomar en cuenta la realidad del estudiante. Por ejemplo, en primera instancia se le presentan situaciones que el alumno pueda ver y tocar (el aula, sus compañeros); después, lo más cercano afectivamente (la familia, los amigos, el pueblo, sus intereses personales, etc.); más tarde, temas de interés típicos de la edad, que sean de actualidad (los juegos tradicionales, las olimpiadas, la vida de los animales, el deporte, etc.). También se pueden incluir algunos temas que permitan relacionar asignaturas entre sí.
3. La clase debe ser abierta, es decir, que permita al alumno decir lo que él desea o introducir temas en la conversación.

4. Deberá incluir una retroalimentación significativa. El profesor, con sus comentarios, gestos, miradas, notas, etc., debe tender a corregir errores de contenido y no de forma.

5. Para el progreso del estudiante es preciso incluir tareas con un nivel de dificultad relacionado con la complejidad lingüística y la comunicativa. Por ejemplo, es más difícil narrar que describir.

6. Es conveniente permitir un período de silencio y un desfase entre comprensión y producción, debido a que la expresión debe surgir lo más espontáneamente posible cuando el alumno haya procesado y organizado el "input".

Para el caso de este proyecto de extensión, se pueden realizar las siguientes actividades con el fin de motivar la expresión oral, las cuales se tomaron en cuenta para los talleres de capacitación: representaciones, juegos de papeles, juegos de descubrimiento, solución de problemas, simulaciones e inventar ejercicios para la clase.

a. Representaciones: Se interpreta frente a la clase un pequeño diálogo. Los alumnos lo interpretan en parejas introduciendo los cambios que se deseen, sustituyendo nombres de países, nacionalidades, ciudades o reinventando un diálogo muy distinto. Cuando lo han practicado lo suficiente, pueden representarlo a la clase. Es importante recalcar que este ejercicio es de producción formal y no de expresión natural.

b. Juegos de papeles: Los estudiantes dan a otros compañeros instrucciones y estos deben conversar con el compañero adoptando el papel asignado. Se pueden crear conversaciones en grupos o bien los alumnos pueden pasear por la clase. Estas actividades deben ser cuidadosamente planificadas por el profesor.

c. Juegos de descubrimiento: Los estudiantes pueden trabajar en parejas o en grupos. Cada uno tiene la información y pueden reconstruir la situación. Por ejemplo, si cada estudiante tiene un dibujo, deben averiguar lo que los diferencia.

d. Solución de problemas: Se presenta algún tipo 
de problema para que los estudiantes busquen la solución. Seguidamente se hace una puesta en común, primero en grupos y después a toda la clase. Los alumnos deben llegar a un acuerdo o saber resumir las diversas posturas.

e. Simulaciones: Cuando el estudiante debe desenvolverse en un contexto imaginario definido por el profesor. Son similares a los juegos de papeles pero la ficción se introduce en la situación y no tanto en la creación de personajes. Se presenta un problema a los alumnos y entre todos se encuentran soluciones.

f. Inventar ejercicios para la clase: Los alumnos pueden crear entrevistas para sus compañeros y ejercicios de comprensión oral, grabados en cintas, para que la clase los escuche y conteste. Después los estudiantes crean pequeñas entrevistas a sus compañeros con tres o cuatro preguntas. Luego graban la entrevista para escucharla y responderla en clase.

Según González (2005), en la búsqueda de alternativas educativas que propicien la motivación del educando por la expresión oral, se considera que la implementación de acciones didácticas usando instrumentos de mediación como el juego y la recreación podría propiciar actividades que promuevan ambientes de aprendizajes ricos en experiencias que en forma amena e interactiva permitan el abordaje de la enseñanza de una destreza lingüística.

El juego y la recreación son oportunidades sociales que tienen una función muy importante en tanto estrategias de atracción y motivación para los educandos. "La infancia y la adolescencia son las etapas de la vida en donde la necesidad y capacidad lúdica alcanzan su más alto nivel de intensidad, la mayor variedad de contenidos, la más clara función de estímulos para el aprendizaje y para una vida social activa" (González, 2005)

Por consiguiente, el taller de capacitación integra una serie de actividades lúdicas que promueven la expresión oral de los niños y las niñas. Es más fácil atraer el interés por los temas mediante actividades recreativas que motiven a los educandos y les brinden la posibilidad de enriquecer su sentido de competencia y autoestima, que mediante actividades puramente académicas.

\section{Desarrollo de los talleres e implementación}

A raíz de la teoría, se decidió plantear cinco sesiones para cada uno de los dos talleres implementados, en los cuales distribuimos las siete escuelas mencionadas. Cada sesión tuvo una duración de cuatro horas diarias, durante el primer semestre de 20 I2. Se plantearon objetivos, contenidos, tiempo, actividades, evaluación y recursos para cada sesión. Se trabajó con grupos de tres personas promedio por escuela. Al final, se le entregó a cada director una carta para firmar un compromiso como institución de darle seguimiento a esta capacitación con los niños y las niñas. Entre los meses de septiembre y octubre de 2012 se visitaron las escuelas para observar el trabajo realizado por los docentes y el resultado que tuvo el proyecto con los alumnos.

La primera sesión consistió en analizar los conceptos relacionados con la comunicación oral, para lograr una mejor comprensión de las técnicas que se requieren en el desarrollo de esta destreza. Se realizaron diagnósticos y grabaciones de los participantes. Hubo lecturas iniciales y ejercicios para una mejor escucha activa. Como tarea, los alumnos debían traer información de un tema que les llamara la atención y buscar en el diccionario todas las palabras desconocidas.

A partir de este primer taller, se detectó una deficiencia en la mayoría de los aspectos de expresión oral de los maestros y maestras de las escuelas visitadas. Para ello se usó una tabla de valoración del diagnóstico, la cual contempla expresión corporal (mirada, gesticulación, postura), expresión oral (dicción, uso de muletillas, volumen, respiración correcta) y contenido de la disertación (estructura del discurso, organización, vocabulario, dominio del tema y reflejo de la práctica previa). Por cada 10 docentes participantes, solamente dos contemplaron mínimo un $70 \%$ de los aspectos necesarios de una buena exposición.

En la segunda sesión, los alumnos tuvieron acceso a la información actualizada de material didáctico para la enseñanza de la expresión oral. Pudieron observar vídeos elaborados por la misma institución (ITCR) y elaboraron un esquema de ideas a partir de la información que trajeron a la clase. Para ello, debieron leer un material específico sobre cómo realizar un discurso, el cual contempla estructura, organización de ideas, objetivo, delimitación del 
tema, paralelismo, tipos de introducción y conclusión, análisis del público y apoyos audiovisuales.

Se detectó en este segundo taller el interés de los educadores por leer sobre un tema al que, según comentaron, no han tenido acceso durante su carrera profesional y, por lo tanto, fue la primera vez que conocieron sobre aspectos tan específicos para la enseñanza de la expresión oral. En este sentido, colaboraron con las actividades, participaron en su totalidad y aclararon dudas sobre las lecturas y vídeos presentados. Se puede decir que un 100\% de los y las participantes se mantuvieron con mucha atención durante todo el taller.

En la tercera sesión se planeó enseñar todos los elementos que deben contemplarse en la expresión corporal al momento de exponer. Por consiguiente, se emplearon estrategias y diferentes consejos para cumplir con el buen uso de los movimientos del cuerpo que acompañan a la oralidad. Se empleó la teoría propuesta por los diversos autores ya mencionados, además de la experiencia de las investigadoras. De igual manera, se concluyó con la revisión del esquema de ideas asignado en la sesión anterior.

La cuarta sesión fue planeada para abarcar cada uno de los aspectos de la elocución. Fue preciso realizar ejercicios de relajación, respiración, vocalización, nasalidad, uso de las vocales y consonantes, pronunciación y volumen. Uno de los ejercicios clave que tuvo un especial impacto en la pronunciación adecuada fue la lectura tonal a través de los textos de diferentes géneros literarios.

En estas dos sesiones, el 100\% de los participantes estuvieron realizando las diversas actividades sin problema alguno. Se notó nuevamente el interés por el tema y la necesidad de aprender para poder implementarlo con sus estudiantes. Fue especial el acompañamiento que hubo por parte de las instructoras del taller, pues debían corregir los errores constantes en el desarrollo de cada una de las actividades y ejercicios programados. Por ejemplo, el lápiz en la boca para pronunciar las palabras y dar cabida al paso del aire por el aparato fonador.

La última sesión se dedicó a aclarar dudas, hacer revisiones finales de algunos de los temas vistos, así como a las presentaciones finales de oratoria de cada uno de los maestros y maestras. Se aclaró que el tiempo requerido para enseñar este taller a los alumnos, epresentaría el doble o triple que el tiempo desempeñado por las investigadoras, debido al grado de comprensión y madurez académica que tienen los maestros a diferencia de sus alumnos. Por tal motivo, fue preciso que los docentes pensaran en cuál sería el tiempo prudente para que sus estudiantes pudieran recibir la teoría y práctica vistas en la capacitación.

Al final de las cinco sesiones, fue satisfactorio observar un cambio positivo en las presentaciones orales. Los maestros requieren, sin duda alguna, de mayor práctica para poder contemplar todos los detalles que debe tener una buena disertación, sin embargo, mostraron cambios importantes, especialmente en su dicción y expresión corporal. De cada diez docentes, siete lograron mejorar en un $80 \%$ el diagnóstico inicial.

\section{Conclusiones y reflexiones a partir de los resultados del estudio}

"Si capturamos una nueva habilidad y conocimiento, y no empleamos ésta o no la potenciamos, será solo parte de una cultura general impráctica (De la Rosa, 2006). La necesidad de compartir el conocimiento que se tiene y la experiencia en el desarrollo de la habilidad oral es la manera de potenciar y crear una cultura de oradores y generadores de conocimiento crítico. Este es el impacto social que se pretende obtener de tal proyecto de extensión.

El objetivo es estimular la habilidad en los maestros y maestras de primaria, así como en los niños y niñas que se preparan para enfrentarse a un futuro incierto en el que hay que defender el criterio y ser críticos de la información que se recibe y se aprende. El primer paso es generar un taller con las mejores estrategias comunicativas que permitan un buen aprendizaje del tema, el segundo es transmitir este conjunto de conocimientos a través de las generaciones para darle posicionamiento a la habilidad oral como una herramienta fundamental que permite ejercer labores profesionales y académicas.

El ser humano es un individuo social, que requiere interactuar para ejercer un papel en sociedad, por lo que la comunicación oral no es una opción, es una necesidad. Este es un mensaje que se imparte a los grupos que reciben la capacitación, porque se quiere provocar una reflexión sobre la verdadera 
importancia de la oratoria moderna para los fines de la educación.

El proyecto provocó impacto en los docentes y estudiantes que lo recibieron porque se pudo observar el fruto de la práctica constante y la dedicación con el fin de obtener un resultado satisfactorio. Los maestros comentaron que impartieron los talleres a los niños en 10 a 15 sesiones de tres horas cada una. Cada tres sesiones realizaron prácticas orales que permitieran incorporar lo aprendido, según lo que se les había enseñado en los talleres iniciales.

Priscilla Gómez (junio 20I2), maestra de la Escuela Filadelfo Salas Céspedes, comentó que "Con el grupo de mis alumnos me ha ayudado porque me puedo expresar mejor con ellos. Yo ya lo he puesto en práctica, les doy un tema para que ellos lo expongan de acuerdo con lo aprendido en el curso".

Un orador es quien logra persuadir con su palabra en la acción, razón o sentimiento del otro. Este concepto se hizo realidad en cada una de las visitas que se realizaron a las escuelas en los meses de septiembre y octubre de 2012. Las investigadoras pudieron observar en cada escuela un grupo de aproximadamente ocho niños por centro educativo, los cuales recibieron la capacitación con sus maestros o maestras. Estos alumnos demostraron su habilidad oral por medio de una disertación sobre temas diversos tales como: derechos humanos, desastres naturales en Costa Rica a través de la historia, la cultura de los cartagos y la belleza natural de su país.

Cada visita fue motivo de satisfacción para las investigadoras al observar los resultados. Por cada grupo de ocho niños, solamente uno no logró expresarse con todas las habilidades comunicativas. Esto quiere decir que el proyecto causó impacto en las escuelas y provocó interés en los educandos. Además, motivó a los centros involucrados a participar en el Concurso de Oratoria de la Municipalidad de Cartago. A diferencia de otros años, hubo un número considerable de participantes que expusieron sobre cultura y tradición costarricense, se notó una mayor identificación con el tema y comunicaron el aporte que darían al país desde su rol como ciudadanos preocupados por el bienestar de Costa Rica.

A continuación se mencionan diversos aspectos que se destacan de los resultados del proyecto en las escuelas, a partir de lo observado en las visitas:
I. Niños y niñas más preocupados por conocer el significado del vocabulario de su discurso.

2. Docentes identificados con la responsabilidad y compromiso de capacitar a sus educandos.

3. Entrega de bitácoras por parte de los docentes sobre el trabajo realizado durante las sesiones que tuvieron con los educandos. Las bitácoras demuestran las actividades realizadas, limitaciones, alcances, conclusiones y observaciones en general. Agregaron fotos de cada sesión.

4. Niños y niñas más interesados por usar su expresión corporal durante la disertación.

5. Mayor dominio del escenario durante la disertación.

6. Los niños y niñas hablaron de los ejercicios de relajación y dicción que aprendieron.

7. La estructura de los discursos cumplió con elementos como: introducción, desarrollo y conclusión, frases de motivación, citas textuales, contenido con un vocabulario adaptado al nivel educativo, exposición a partir de un esquema de ideas y análisis del público.

8. Como mínimo, un estudiante representó a su escuela en el Concurso de Oratoria de la Municipalidad de Cartago.

9. Los directores de las escuelas y los maestros destacaron el agradecimiento al ITCR por incluirlos en este proyecto de extensión y propusieron una segunda etapa para continuar con los talleres.

\section{Bibliografía}

Bello, P. et al. (1990). Didáctica de las Segundas Lenguas. Estrategias y Recursos Básicos. Aula XXI. Madrid: Santillana.

Bradley, B.E. (1984). Fundamentals of Speech Communication. $4^{\mathrm{a}}$ ed. EE.UU: Brown Publisher.

Brehler, R. (2000). Prácticas de oratoria moderna. 2 ed. Madrid: Editorial El Drac.

Briz, A. (2008). Saber hablar. México: Editorial Santillana.

Carnegie, D. (1990). El camino fácil y rápido para hablar eficazmente. Argentina: Editorial Sudamericana.

Catta, R. (1982). Cómo hablar en público. 2 ed. Bilbao: Colección Mensajero.

Cuervo, M. \& Jesús, D. (1998). Mejorar la expresión oral. Madrid: Narcea De Ediciones. 
De la Rosa, C. (2006). Oratoria motivacional. Enciclopedia de Oratoria Motivacional. Primera edición digital 2006. Material de divulgación cultural.

Fonseca, Y. (2005). Comunicación oral. Fundamentos y práctica estratégica. México: Pearson Educación.

Fournier, C. (2005). Comunicación verbal. México: International Thompson Editores.

Gónzález, F. (2005). Guía metodológica para la enseñanza de la morfología y la sintaxis en el cuarto ciclo de la Educación secundaria. Informe final del proyecto para optar por el grado de Magister en Planificación Curricular. Universidad de Costa Rica.

Grzybowski, G. (1978). Saber improvisar. Unas palabras, un escrito, una acción. Bilbao: Mensajero.

McGraw-Hill. (2009). La comunicación oral. Punto 02. Material extraído 28 el agosto 20 I2. Disponible en: www.mcgrawhill. es/bcv/guide/capitulo/8448 I7| I52.pdf.

Martínez, J. (200 I). Aprender a comunicarse en público. Barcelona: Editorial Paidós.

McEntee, E. (2004). Comunicación oral. 2 ed. México: McGraw Hill/Interamericana Editores.
Muller, M. (1989). Técnicas de comunicación oral. San José: Editorial Universidad de Costa Rica.

Prochnow, V. (1983). Tratado de oratoria. El arte de expresarse. México: Editorial Continental.

Richards, C. (1990). The Language Teaching Matrix. EE.UU: University of Cambridge.

Spitzberg, B.H. (2003). Methods of interpersonal skill assessment. En Greene, J.O., Burleson, B.R. (Eds.). Handbook of interpersonal communication. Sage:Thousand Oaks, CA.

Urpí, M. (2004). Aprender comunicación no verbal. La elocuencia del silencio. Barcelona: Ediciones Paidós Ibérica.

Vallarta, L. (1983). La oratoria al alcance de todos. México: Carlos Césarman.

Verderber, R. (1999). Comunícate. 9 ed. México: International Thompson Editores.

Verderber, R. (2000). Comunicación oral efectiva. II ed. México: International Thompson Editores.

Zacharis, J., Bender, C. (1978). Comunicación oral. Un enfoque racional. México: LIMUSA. 